TUM/T39-02-01

\title{
Chiral dynamics of nuclear matter at finite temperature $\phi^{\dagger}$
}

\author{
S. Fritsch ${ }^{a}$, N. Kaiser ${ }^{a}$ and W. Weise ${ }^{a, b}$ \\ ${ }^{a}$ Physik Department, Technische Universität München, D-85747 Garching, Germany \\ ${ }^{b}$ ECT*$^{*}$, I-38050 Villazzano (Trento), Italy \\ email: nkaiser@physik.tu-muenchen.de
}

\begin{abstract}
We extend a recent three-loop calculation of nuclear matter in the systematic framework of chiral perturbation theory to finite temperatures $T$. The contributions from oneand two-pion exchange diagrams which cause nuclear binding and saturation at $T=0$ are included for $T>0$ in the density and temperature dependent free energy per particle, $\bar{F}(\rho, T)$. The so-called anomalous $2 \pi$-exchange contribution $\bar{A}(\rho, T)$ (with no counterpart in the ground state energy density at $T=0$ ) is consistently included. The calculated pressure isotherms display the familiar first-order liquid-gas phase transition of isospin symmetric nuclear matter with a critical point at $T_{c}=25.5 \mathrm{MeV}$ and $\rho_{c}=0.09 \mathrm{fm}^{-3}$. The too high value of the critical temperature originates from the strong momentum dependence of the underlying single-particle potential $U\left(p, k_{f 0}\right)$ near the Fermi-surface. We also consider pure neutron matter at $T>0$ in the same framework and find fair agreement with sophisticated many-body calculations for neutron densities $\rho_{n}<0.2 \mathrm{fm}^{-3}$.
\end{abstract}

PACS: 12.38.Bx, 21.65.+f

Keywords: Effective field theory at finite density and temperature; Liquid-gas phase transition of nuclear matter; Neutron matter at finite temperature.

The analysis of data from low-energy heavy-ion collisions in the regime of nuclear fragmentation has lead to the picture that heated nuclear matter undergoes a first-order phase transition from a liquid-like state to a vaporized gas state [1, 2]. This liquid-gas phase transition of isospin symmetric nuclear matter is in fact very similar to that of the familiar van-der-Waals gas, with a corresponding critical temperature of $T_{c} \simeq(16-18) \mathrm{MeV}$ [2] and a critical density of $\rho_{c}=(0.06-0.07) \mathrm{fm}^{-3}$ [2]. Clearly, the dynamical description of this phase transition is an important topic in any microscopic calculation of nuclear matter. In the $\sigma \omega$-mean field model of Serot and Walecka [3] nucleons are described as Dirac-quasiparticles moving in self-consistently generated scalar and vector mean fields and a critical temperature of $T_{c} \simeq 19 \mathrm{MeV}$ is typically found [ [. As an other example, the sophisticated many-body calculations of the Urbana group [5] using the V14 effective NN-interaction (plus an adjustable three-nucleon interaction) predict a critical temperature of $T_{c} \simeq 18 \mathrm{MeV}$ [5]. For earlier work on this topic using effective Skyrme forces, see ref.[6].

In a recent work [7], we have used chiral perturbation theory for a systematic treatment of the nuclear matter many-body problem. In this calculation the contributions to the energy per particle, $\bar{E}\left(k_{f}\right)$, originate exclusively from one- and two-pion exchange between nucleons and they are ordered in powers of the Fermi momentum $k_{f}$ (modulo functions of $k_{f} / m_{\pi}$ ). It

\footnotetext{
${ }^{1}$ Work supported in part by BMBF, GSI and DFG.
} 
has been demonstrated in ref. [7] that the empirical saturation point and the nuclear matter compressibility $K \simeq 255 \mathrm{MeV}$ can be well reproduced at order $\mathcal{O}\left(k_{f}^{5}\right)$ in the chiral expansion with just one single momentum cut-off scale of $\Lambda \simeq 0.65 \mathrm{GeV}$ which parameterizes the necessary short range dynamics. Most surprisingly, the prediction for the asymmetry energy, $A\left(k_{f 0}\right)=$ $33.8 \mathrm{MeV}$, is in very good agreement with its empirical value. Furthermore, as a nontrivial fact pure neutron matter is predicted to be unbound and the corresponding equation of state agrees roughly with that of sophisticated many-body calculations for low neutron densities $\rho_{n} \leq$ $0.25 \mathrm{fm}^{-3}$. In a subsequent work [8], the (complex-valued) momentum and density dependent single-particle potential $U\left(p, k_{f}\right)+i W\left(p, k_{f}\right)$ (i.e. the average nuclear mean field) has been calculated in the same framework. It was found that chiral $1 \pi$ - and $2 \pi$-exchange give rise to a potential depth for a nucleon at the bottom of the Fermi sea of $U\left(0, k_{f 0}\right)=-53.2 \mathrm{MeV}$. This value is in very good agreement with the depth of the empirical optical model potential and the nuclear shell model potential.

Given the fact that many properties of nuclear matter can be well described by chiral $\pi N$ dynamics treated (perturbatively) up to three loop order it is natural to consider in a further step finite temperatures $T$ in order to check whether the first-order liquid-gas phase transition of nuclear matter is correctly reproduced by this particular dynamics. Such an investigation is the subject of the present paper.

For the relatively low temperatures $T \leq 30 \mathrm{MeV}$ which are of interest in this context one can safely neglect effects from thermal pions or thermally excited nucleon-antinucleon pairs. As a consequence, nucleons can be treated non-relativistically and the new parameter, the temperature $T$, enters only through the nucleons' thermal occupation probabilities given by a Fermi-Dirac distribution.

In ref.[7] the diagrammatic calculation of the energy density at $T=0$ (as a function of the particle density $\rho$ ) has been organized in the number of so-called medium insertions. The latter is a technical notation for the difference between the in-medium and vacuum nucleon propagator (see eq.(3) in ref.[7]). A thermodynamically consistent extension of such an ordering scheme to finite temperatures is to relate it directly to the free energy density $\rho \bar{F}(\rho, T)$, since its natural thermodynamical variables are the particle density $\rho$ and the temperature $T$. In that case the free energy density of isospin symmetric nuclear matter consists of a sum of convolution integrals of the form,

$$
\begin{aligned}
\rho \bar{F}(\rho, T)= & 4 \int_{0}^{\infty} \mathrm{d} p_{1} p_{1} \mathcal{K}_{1} d\left(p_{1}\right)+\int_{0}^{\infty} \mathrm{d} p_{1} \int_{0}^{\infty} \mathrm{d} p_{2} \mathcal{K}_{2} d\left(p_{1}\right) d\left(p_{2}\right) \\
& +\int_{0}^{\infty} \mathrm{d} p_{1} \int_{0}^{\infty} \mathrm{d} p_{2} \int_{0}^{\infty} \mathrm{d} p_{3} \mathcal{K}_{3} d\left(p_{1}\right) d\left(p_{2}\right) d\left(p_{3}\right)+\rho \overline{\mathcal{A}}(\rho, T),
\end{aligned}
$$

with the corresponding kernels $\mathcal{K}_{j}$ and the anomalous contribution $\overline{\mathcal{A}}(\rho, T)$ to be specified.2

\footnotetext{
${ }^{2}$ Let us briefly motivate our approach. The standard procedure in field theory is to calculate the grand canonical partition function $Z$ or equivalently the thermodynamic potential $\Omega(\mu, T)=-(T / V) \ln Z$ as a function of its natural variables, the (non-relativistic) chemical potential $\mu$ and the temperature $T$. In the case of nuclear matter at $T=0$ the functional relationship $\Omega(\mu, 0)$ is however double-valued for $\mu \leq 0$. Since perturbation theory usually gives single-valued thermodynamic functions it is more appropriate to calculate first the (singlevalued) energy density $\rho \bar{E}\left(k_{f}\right)$ of nuclear matter as a function of the particle density $\rho$, as done e.g. in refs. [7, 9]. Then eq.(1) defines a thermodynamically consistent extension to finite temperatures $T$, with the correct $T=0$ limit. This calculational scheme can also be understood such that in the Legendre-transformation from $\Omega(\mu, T)$ to the free energy density, $\rho \bar{F}(\rho, T)=\Omega(\mu, T)-\mu \partial \Omega / \partial \mu$, the derivative term is taken into account only for the non-interacting (free nucleon gas) part and the (static) one-pion exchange contribution, but not for the higher order contributions coming from chiral $2 \pi$-exchange.
} 

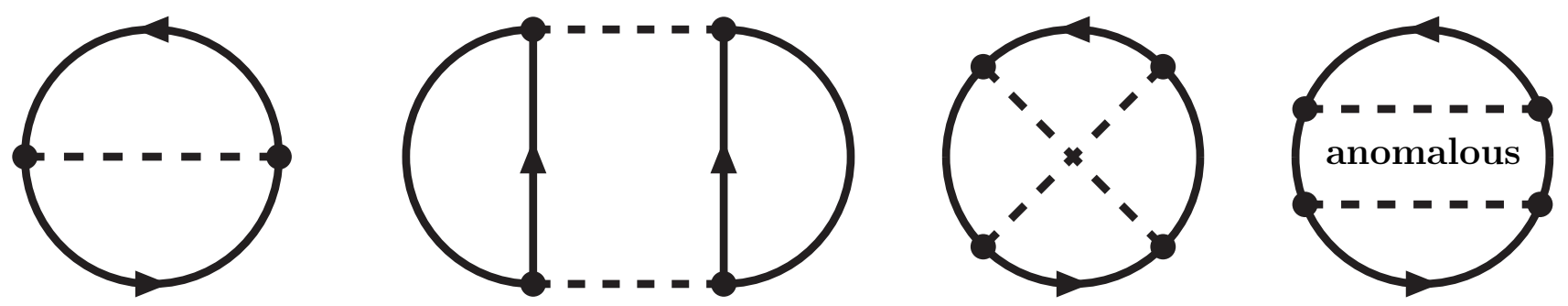

Figure 1: The one-pion exchange Fock-diagram, the iterated one-pion exchange Hartree- and Fock-diagrams and the anomalous Fock-diagram. The combinatoric factors of these diagrams are $1 / 2,1 / 4,1 / 4$ and $1 / 2$, in the order shown.

The quantity

$$
d\left(p_{j}\right)=\frac{p_{j}}{2 \pi^{2}}\left[1+\exp \frac{p_{j}^{2}-2 M \tilde{\mu}}{2 M T}\right]^{-1},
$$

denotes the density of nucleon states in momentum space. It is the product of the temperature dependent Fermi-Dirac distribution and a kinematical prefactor $p_{j} / 2 \pi^{2}$ which has been included in $d\left(p_{j}\right)$ for convenience. $M=939 \mathrm{MeV}$ stands for the (free) nucleon mass. The particle density $\rho$ is calculated from the density of states in momentum space as

$$
\rho=4 \int_{0}^{\infty} \mathrm{d} p_{1} p_{1} d\left(p_{1}\right)=-\sqrt{2}\left(\frac{M T}{\pi}\right)^{3 / 2} \operatorname{Li}_{3 / 2}\left(-e^{\tilde{\mu} / T}\right),
$$

and this relationship determines the dependence of the effective one-body "chemical potential" $\tilde{\mu}(\rho, T)$ on the thermodynamical variables $\rho$ and $T$. The "true" chemical potential is different and given by the formula $\mu=\bar{F}+\rho \partial \bar{F} / \partial \rho$. Concerning eqs. $(1,2,3)$ we are following here (partially) the approximation scheme of ref. 10. Our approach is (by construction) thermodynamically consistent, since all thermodynamic quantities are derived (via standard relations) from the free energy density $\rho \bar{F}(\rho, T)$. The infinite series $\operatorname{Li}_{\nu}(x)=\sum_{k=1}^{\infty} k^{-\nu} x^{k}$ defines the polylogarithmic function of index $\nu$ for $|x|<1$.

The one-body kernel $\mathcal{K}_{1}$ in eq.(1) represents the contribution of the non-interacting nucleon gas to the free energy density and it reads

$$
\mathcal{K}_{1}=\tilde{\mu}-\frac{p_{1}^{2}}{3 M}-\frac{p_{1}^{4}}{8 M^{3}} .
$$

While the first two terms are standard [10], the correction term, $-p_{1}^{4} / 8 M^{3}$, has been constructed according to the following criteria. First, it ensures the correct $T=0$ limit for the energy per particle $\bar{E}\left(k_{f}\right)$ up to order $k_{f}^{4}$, in which $\tilde{\mu}(\rho, 0)=k_{f}^{2} / 2 M$ and $\rho=2 k_{f}^{3} / 3 \pi^{2}$. Secondly, the soconstructed kernel $\mathcal{K}_{1}$ combined with the non-relativistic Fermi-Dirac distribution (see eq.(2)) gives a very accurate approximation of the free energy density of a fully relativistic free nucleon gas [4] for the densities and temperatures of interest here. The factor 4 in eqs. $(1,3)$ counts the spin-isospin multiplicity of a nucleon.

The (non-anomalous) contributions to the free energy density $\rho \bar{F}(\rho, T)$ arising from pionexchange interactions are encoded in the kernels $\mathcal{K}_{2,3}$ in eq.(1). The closed vacuum diagrams related to one-pion exchange (Fock-diagram) and iterated one-pion exchange (Hartree- and 
Fock-diagrams) are shown in Fig. 1. The $1 \pi$-exchange Fock-diagram including the relativistic $1 / M^{2}$-correction leads to the following contribution to the two-body kernel $\mathcal{K}_{2}$ :

$$
\begin{aligned}
\mathcal{K}_{2}^{(1 \pi)}= & \frac{3 g_{A}^{2}}{16 f_{\pi}^{2}}\left\{8 p_{1} p_{2}-2 m_{\pi}^{2} \ln \frac{m_{\pi}^{2}+\left(p_{1}+p_{2}\right)^{2}}{m_{\pi}^{2}+\left(p_{1}-p_{2}\right)^{2}}+\frac{1}{M^{2}}\left[-4 p_{1} p_{2}\left(p_{1}^{2}+p_{2}^{2}\right)\right.\right. \\
& \left.\left.+m_{\pi}^{2}\left(p_{1}^{2}+p_{2}^{2}\right) \ln \frac{m_{\pi}^{2}+\left(p_{1}+p_{2}\right)^{2}}{m_{\pi}^{2}+\left(p_{1}-p_{2}\right)^{2}}-\frac{2 m_{\pi}^{2} p_{1} p_{2}\left(p_{1}^{2}-p_{2}^{2}\right)^{2}}{\left[m_{\pi}^{2}+\left(p_{1}+p_{2}\right)^{2}\right]\left[m_{\pi}^{2}+\left(p_{1}-p_{2}\right)^{2}\right]}\right]\right\} .
\end{aligned}
$$

As in ref. [7], we choose the value $g_{A}=1.3$ for the nucleon axial vector coupling constant, $f_{\pi}=$ 92.4 MeV denotes the weak pion decay constant and $m_{\pi}=135 \mathrm{MeV}$ stands for the (neutral) pion mass. The iterated $1 \pi$-exchange Hartree-graph (second diagram in Fig. 1) contributes to the two-body kernel $\mathcal{K}_{2}$ with the term:

$$
\begin{aligned}
\mathcal{K}_{2}^{(H)}= & \frac{3 g_{A}^{4} M m_{\pi}^{2}}{8 \pi f_{\pi}^{4}}\left\{\left(p_{1}+p_{2}\right) \arctan \frac{p_{1}+p_{2}}{m_{\pi}}\right. \\
& \left.+\left(p_{2}-p_{1}\right) \arctan \frac{p_{1}-p_{2}}{m_{\pi}}-\frac{5}{8} m_{\pi} \ln \frac{m_{\pi}^{2}+\left(p_{1}+p_{2}\right)^{2}}{m_{\pi}^{2}+\left(p_{1}-p_{2}\right)^{2}}\right\} .
\end{aligned}
$$

In this expression we have omitted the contribution of a linear divergence proportional to the momentum cut-off $\Lambda$ (see ref. [7]). All such powerlike terms in $\Lambda$ are collected in eq.(10). Similarly, the iterated $1 \pi$-exchange Fock-graph (third diagram in Fig. 1) gives rise to a two-body kernel $\mathcal{K}_{2}$ of the form:

$$
\begin{aligned}
\mathcal{K}_{2}^{(F)}= & \frac{3 g_{A}^{4} M m_{\pi}}{32 \pi f_{\pi}^{4}}\left\{2 p_{1} p_{2}+m_{\pi}^{2} \int_{\left|p_{1}-p_{2}\right| / 2 m_{\pi}}^{\left(p_{1}+p_{2}\right) / 2 m_{\pi}} \frac{\mathrm{d} x}{1+2 x^{2}}\right. \\
& \left.\times\left[\left(1+8 x^{2}+8 x^{4}\right) \arctan x-\left(1+4 x^{2}\right) \arctan 2 x\right]\right\} .
\end{aligned}
$$

The additional diagrams of irreducible $2 \pi$-exchange (not shown here, but see Fig. 4 in ref. [7]) generate a contribution to the two-body kernel $\mathcal{K}_{2}$ via the expression

$$
\mathcal{K}_{2}^{(2 \pi)}=\frac{m_{\pi}^{4}}{128 \pi^{2} f_{\pi}^{4}}\left\{I\left(\frac{p_{1}+p_{2}}{2 m_{\pi}}\right)-I\left(\frac{\left|p_{1}-p_{2}\right|}{2 m_{\pi}}\right)\right\},
$$

with the function

$$
\begin{aligned}
I(x)= & 3\left(11 g_{A}^{4}-2 g_{A}^{2}-1\right) \ln ^{2}\left(x+\sqrt{1+x^{2}}\right) \\
& +2\left(g_{A}^{2}-1\right)\left[g_{A}^{2}\left(31+22 x^{2}\right)+5+2 x^{2}\right] x \sqrt{1+x^{2}} \ln \left(x+\sqrt{1+x^{2}}\right) \\
& +\left(7-2 g_{A}^{2}+91 g_{A}^{4}\right) x^{2}+\left(3+14 g_{A}^{2}-g_{A}^{4}\right) x^{4} \\
& +\left[12\left(15 g_{A}^{4}-6 g_{A}^{2}-1\right) x^{2}+4\left(11 g_{A}^{4}-10 g_{A}^{2}-1\right) x^{4}\right] \ln \frac{m_{\pi}}{2 \Lambda},
\end{aligned}
$$

obtained from solving the pion-loop integrals. The complete expression for the power divergences specific to cut-off regularization reads:

$$
\mathcal{K}_{2}^{(\Lambda)}=\frac{3 \Lambda p_{1} p_{2}}{32 \pi^{2} f_{\pi}^{4}}\left[-10 g_{A}^{4} M+\left(3 g_{A}^{2}+1\right)\left(g_{A}^{2}-1\right) \Lambda\right]
$$

The term linear in the cut-off $\Lambda$ stems from iterated $1 \pi$-exchange with a contribution of the Hartree- and Fock diagram in the ratio $4: 1$. The term quadratic in the cut-off, on the other hand, originates from irreducible $2 \pi$-exchange. Note that the kernel $\mathcal{K}_{2}^{(\Lambda)}$ in eq.(10) leads to a 
temperature independent contribution to free energy per particle $\bar{F}(\rho, T) \sim \rho$. Therefore it is fully equivalent to a momentum independent NN-contact interaction.

Next, we come to the three-body kernel $\mathcal{K}_{3}$ which incorporates the temperature and density dependent Pauli-blocking effects in intermediate NN-states. The iterated $1 \pi$-exchange Hartreegraph contributes to the three-body kernel $\mathcal{K}_{3}$ in the form:

$$
\mathcal{K}_{3}^{(H)}=\frac{3 g_{A}^{4} M}{4 f_{\pi}^{4}} \int_{\left|p_{1}-p_{2}\right|}^{p_{1}+p_{2}} \mathrm{~d} q \frac{q^{4}}{\left(m_{\pi}^{2}+q^{2}\right)^{2}} \ln \frac{\left|p_{1}^{2}-p_{2}^{2}+q^{2}+2 p_{3} q\right|}{\left|p_{1}^{2}-p_{2}^{2}+q^{2}-2 p_{3} q\right|}
$$

and from the iterated $1 \pi$-exchange Fock-graph one finds,

$$
\begin{aligned}
\mathcal{K}_{3}^{(F)=} & \frac{3 g_{A}^{4} M}{16 f_{\pi}^{4}}\left\{\frac{1}{8 p_{3}^{3}}\left[4 p_{1} p_{3}+\left(p_{3}^{2}-p_{1}^{2}-m_{\pi}^{2}\right) \ln \frac{m_{\pi}^{2}+\left(p_{1}+p_{3}\right)^{2}}{m_{\pi}^{2}+\left(p_{1}-p_{3}\right)^{2}}\right]\right. \\
& \times\left[4 p_{2} p_{3}+\left(p_{3}^{2}-p_{2}^{2}-m_{\pi}^{2}\right) \ln \frac{m_{\pi}^{2}+\left(p_{2}+p_{3}\right)^{2}}{m_{\pi}^{2}+\left(p_{2}-p_{3}\right)^{2}}\right] \\
& \left.+\int_{\left|p_{2}-p_{3}\right|}^{p_{2}+p_{3}} \mathrm{~d} q \frac{q^{2}}{m_{\pi}^{2}+q^{2}}\left[\ln \frac{\left|p_{1}+h\right|}{\left|p_{1}-h\right|}+\frac{m_{\pi}^{2}}{R} \ln \frac{\left|p_{1} R+\left(p_{1}^{2}-p_{3}^{2}-m_{\pi}^{2}\right) h\right|}{\left|p_{1} R+\left(p_{3}^{2}+m_{\pi}^{2}-p_{1}^{2}\right) h\right|}\right]\right\},
\end{aligned}
$$

with the abbreviations:

$$
R=\sqrt{\left(m_{\pi}^{2}+p_{1}^{2}-p_{3}^{2}\right)^{2}+4 m_{\pi}^{2}\left(p_{3}^{2}-h^{2}\right)}, \quad h=\frac{1}{2 q}\left(p_{2}^{2}-p_{3}^{2}-q^{2}\right) .
$$

Note that all integrands in representations of $\mathcal{K}_{2,3}$ are odd functions of their respective integration variable and therefore one could even drop the absolute magnitude on the lower integration limits. We also remind that (non-anomalous) terms involving the product of four Fermi-Dirac distributions are effectively absent in second order perturbation theory [1]. Because of the antisymmetry of the accompanying energy denominator under the exchange of two pairs of momenta these terms integrate to zero.

Next, we come to the so-called anomalous contribution $\overline{\mathcal{A}}(\rho, T)$ in eq.(1). This is a special feature at finite temperatures [11, 12] with no counterpart in the calculation of the ground state energy density $\rho \bar{E}\left(k_{f}\right)$ at $T=0$. From the "anomalous" Fock-diagram in Fig. 1 we derive the following contribution to the free energy per particle of isospin symmetric nuclear matter:

$$
\begin{aligned}
\overline{\mathcal{A}}(\rho, T)= & -\frac{\left[\Omega_{1 \pi}^{\prime}(\rho, T)\right]^{2}}{2 \rho \Omega_{0}^{\prime \prime}(\rho, T)} \\
& +\frac{9 g_{A}^{4}}{8 f_{\pi}^{4} T \rho} \int_{0}^{\infty} \mathrm{d} p_{1} \int_{0}^{\infty} \mathrm{d} p_{2} \int_{0}^{\infty} \mathrm{d} p_{3} d\left(p_{1}\right) d\left(p_{2}\right)\left[2 \pi^{2} d\left(p_{2}\right)-p_{2}\right] d\left(p_{3}\right) \\
& \times\left[p_{1}-\frac{m_{\pi}^{2}}{4 p_{2}} \ln \frac{m_{\pi}^{2}+\left(p_{1}+p_{2}\right)^{2}}{m_{\pi}^{2}+\left(p_{1}-p_{2}\right)^{2}}\right]\left[p_{3}-\frac{m_{\pi}^{2}}{4 p_{2}} \ln \frac{m_{\pi}^{2}+\left(p_{3}+p_{2}\right)^{2}}{m_{\pi}^{2}+\left(p_{3}-p_{2}\right)^{2}}\right]
\end{aligned}
$$

with the $\tilde{\mu}$-derivative of the thermodynamical potential due to static $1 \pi$-exchange,

$$
\Omega_{1 \pi}^{\prime}(\rho, T)=\frac{3 g_{A}^{2} M}{2 f_{\pi}^{2}} \int_{0}^{\infty} \mathrm{d} p_{1} \int_{0}^{\infty} \mathrm{d} p_{2} d\left(p_{1}\right) \frac{d\left(p_{2}\right)}{p_{2}}\left[\frac{\left(p_{1}+p_{2}\right)^{3}}{m_{\pi}^{2}+\left(p_{1}+p_{2}\right)^{2}}+\frac{\left(p_{1}-p_{2}\right)^{3}}{m_{\pi}^{2}+\left(p_{1}-p_{2}\right)^{2}}\right],
$$

and the second $\tilde{\mu}$-derivative of the free nucleon gas part,

$$
\Omega_{0}^{\prime \prime}(\rho, T)=-4 M \int_{0}^{\infty} \mathrm{d} p_{1} \frac{d\left(p_{1}\right)}{p_{1}}=\sqrt{2 T}\left(\frac{M}{\pi}\right)^{3 / 2} \operatorname{Li}_{1 / 2}\left(-e^{\tilde{\mu} / T}\right)
$$


The first term in eq.(14) originates from taking into account the (static) $1 \pi$-exchange contribution in the Legendre-transformation from the thermodynamical potential to the free energy density and from the perturbative shift of the chemical potential $\tilde{\mu} \rightarrow \tilde{\mu}-\Omega_{1 \pi}^{\prime}(\rho, T) / \Omega_{0}^{\prime \prime}(\rho, T)$ (for details on that procedure, see ref. [12]). We have explicitly checked that the anomalous contribution $\overline{\mathcal{A}}(\rho, T)$ vanishes identically at $T=0$ for all densities $\rho$. This vanishing, $\overline{\mathcal{A}}(\rho, 0)=0$, is an automatic consequence of the Kohn-Luttinger-Ward theorem [12] which of course holds in our case since the Fermi-surface and the pion-induced interactions are invariant under spatial rotations. Furthermore, it is interesting to observe that the temperature and density dependent anomalous contribution $\overline{\mathcal{A}}(\rho, T)$ vanishes identically in the chiral limit $m_{\pi}=0$. Note also that the anomalous contribution $\overline{\mathcal{A}}(\rho, T)$ is (formally) of the same order in small momenta as the contributions to $\bar{F}(\rho, T)$ coming from iterated $1 \pi$-exchange. It must therefore not be neglected in a consistent and complete calculation.

Via general thermodynamical relations [4] one finally derives from the free energy per particle $\bar{F}(\rho, T)$ the pressure $P(\rho, T)$ and the entropy per particle $\bar{S}(\rho, T)$ as:

$$
P(\rho, T)=\rho^{2} \frac{\partial \bar{F}(\rho, T)}{\partial \rho}, \quad \bar{S}(\rho, T)=-\frac{\partial \bar{F}(\rho, T)}{\partial T}
$$

We are now in the position to present numerical results for isospin symmetric nuclear matter at finite temperatures. We use consistently the same parameters as in our previous work [7]. There, our only parameter, the cut-off scale $\Lambda=646.3 \mathrm{MeV} \simeq 7 f_{\pi}$, has been fine-tuned to the binding energy per particle, $-\bar{E}\left(k_{f 0}\right)=15.26 \mathrm{MeV}$. Fig. 2 shows the free energy per particle $\bar{F}(\rho, T)$ as a function of the nucleon density $\rho$ for various temperatures $T=0,10,18,25.5,30 \mathrm{MeV}$. The uppermost line is the attractive branch of the nuclear matter saturation curve at $T=0$. The singular behavior of the free energy per particle $\bar{F}(\rho, T)$ for $\rho \rightarrow 0$ at $T>0$ is a generic feature (see e.g. the figures and tables corresponding to the results of the Urbana group in ref. [5]). The internal energy per particle $\bar{F}(\rho, T)+T \bar{S}(\rho, T)$, on the other hand, approaches approximately the value $3 T / 2$ for $\rho \rightarrow 0$.

Fig. 3 shows the calculated pressure isotherms $P(\rho, T)$ of isospin symmetric nuclear matter. As it should be these curves display a first-order liquid-gas phase transition similar to that of the van-der-Waals gas. The coexistence region between the liquid and the gas phase (which has to be determined by the Maxwell construction [4]) terminates at the critical temperature $T_{c}$. From there on the pressure isotherms $P(\rho, T)$ grow monotonically with the particle density $\rho$. In the present calculation we find a critical temperature of $T_{c}=25.5 \mathrm{MeV}$ and a critical density of $\rho_{c}=0.09 \mathrm{fm}^{-3} \simeq 0.5 \rho_{0}$ (with $\rho_{0}=0.178 \mathrm{fm}^{-3}$, the predicted nuclear matter saturation density). Together with the critical pressure $P\left(\rho_{c}, T_{c}\right)=0.69 \mathrm{MeV} \mathrm{fm}^{-3}$ this prediction of the critical point deviates considerably from most other calculations. The too high value of the critical temperature $T_{c}=25.5 \mathrm{MeV}$ finds its explanation in the strong momentum dependence of the underlying single-particle potential $U\left(p, k_{f 0}\right)$ near the Fermi-surface $p=k_{f 0}$ (see Fig. 3 in ref. [8]). The nominal value of the effective nucleon mass at the Fermi-surface is $M^{*}\left(k_{f 0}\right) \simeq 3 M$. The latter quantity determines via the reciprocally related density of states at the Fermisurface completely the low-temperature behavior of a Fermi-liquid. Since in our calculation the density of thermally excitable quasi-particles is too low nuclear matter has to be heated up to higher temperatures until it evaporates completely. More elaborate calculations of nuclear matter in effective (chiral) field theory are necessary in order to cure this particular problem of the large effective nucleon mass $M^{*}\left(k_{f 0}\right)$. Note that despite its large isospin factor 18 the anomalous contribution $\overline{\mathcal{A}}(\rho, T)$ does practically not influence the behavior of nuclear matter at low temperatures $T<30 \mathrm{MeV}$.

As a side remark, we consider the chiral limit $m_{\pi}=0$. With a reduced cut-off scale of $\Lambda=$ $555.8 \mathrm{MeV}$ (and fixed $\left.g_{A}, f_{\pi}, M\right)$ the same maximum binding energy per particle $(15.26 \mathrm{MeV})$ is 


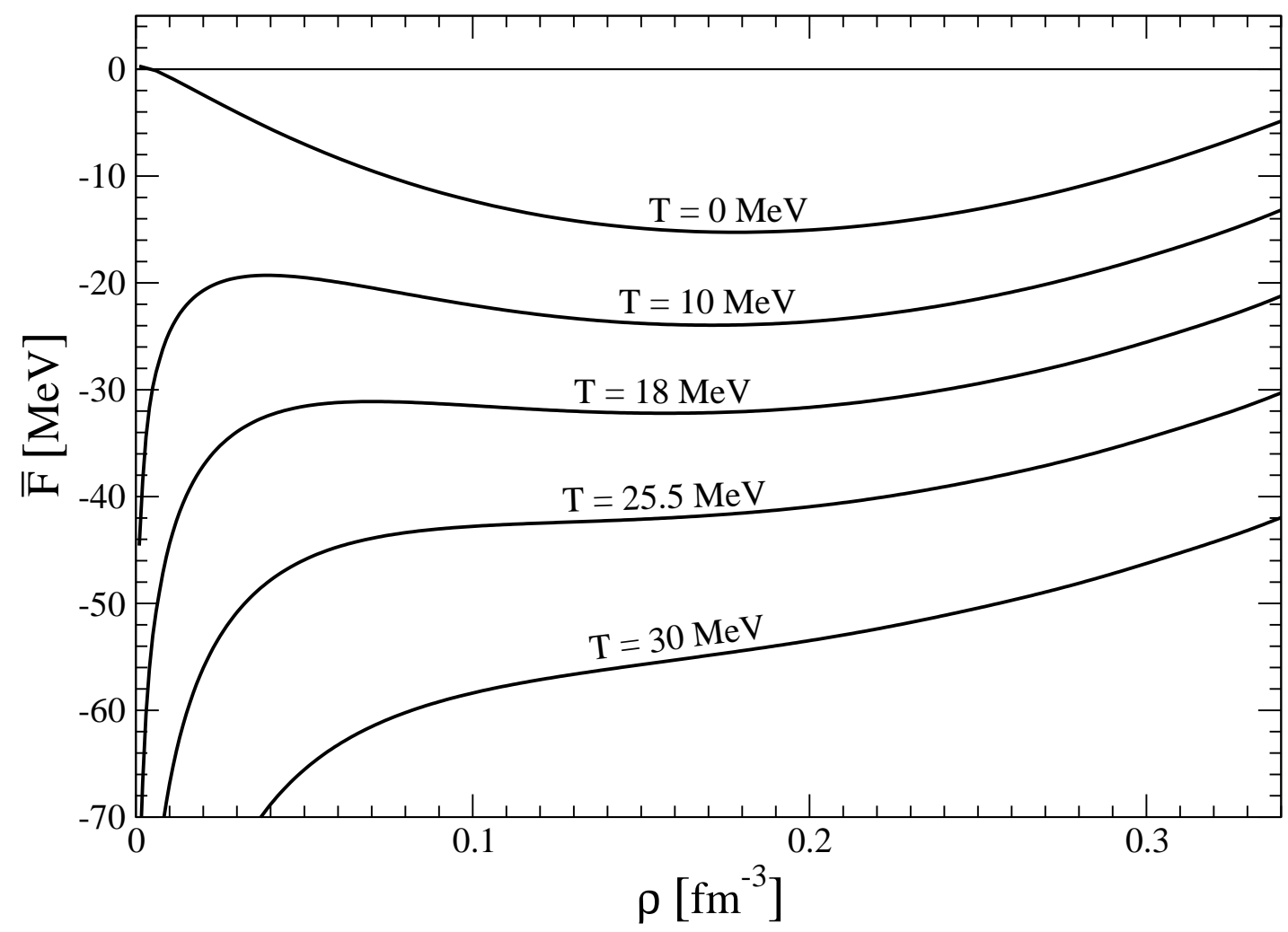

Figure 2: The free energy per particle of isospin symmetric nuclear matter $\bar{F}(\rho, T)$ versus the nucleon density $\rho$. Each curve is labeled with its corresponding constant temperature $T$.

obtained at a saturation density of $\rho_{0}=0.145 \mathrm{fm}^{-3}$. Interestingly, the critical temperature $T_{c} \simeq$ $27 \mathrm{MeV}$ remains nearly unchanged when taking the chiral limit. This confirms the expectation that the critical temperature $T_{c}$ is primarily determined by the binding energy per particle $-\bar{E}\left(k_{f 0}\right)$ and the effective nucleon mass at the Fermi-surface $M^{*}\left(k_{f 0}\right)$.

The present calculation is readily adapted to the case of pure neutron matter at finite temperatures $T$. Only the isospin factors of the pion-exchange diagrams (see Fig. 1) change when switching from isospin symmetric nuclear matter to pure neutron matter. The free energy per neutron $\bar{F}_{n}\left(\rho_{n}, T\right)$ takes a form analogous to eq.(1), namely:

$$
\begin{aligned}
\rho_{n} \bar{F}_{n}\left(\rho_{n}, T\right)= & 2 \int_{0}^{\infty} \mathrm{d} p_{1} p_{1} \mathcal{K}_{1} d\left(p_{1}\right)+\int_{0}^{\infty} \mathrm{d} p_{1} \int_{0}^{\infty} \mathrm{d} p_{2} \mathcal{K}_{n, 2} d\left(p_{1}\right) d\left(p_{2}\right) \\
& +\int_{0}^{\infty} \mathrm{d} p_{1} \int_{0}^{\infty} \mathrm{d} p_{2} \int_{0}^{\infty} \mathrm{d} p_{3} \mathcal{K}_{n, 3} d\left(p_{1}\right) d\left(p_{2}\right) d\left(p_{3}\right)+\rho_{n} \overline{\mathcal{A}}_{n}\left(\rho_{n}, T\right) .
\end{aligned}
$$

The neutron density $\rho_{n}=\rho / 2$ is reduced by a factor 2 in comparison to $\rho$ calculated via eq.(3) and the same applies to the one-body term proportional to $\mathcal{K}_{1}$ in eq.(18). The contributions from $1 \pi$-exchange and iterated $1 \pi$-exchange to the neutron kernels $\mathcal{K}_{n, j}$ are determined by certain relative isospin factors as,

$$
\mathcal{K}_{n, 2}^{(1 \pi)}=\frac{1}{6} \mathcal{K}_{2}^{(1 \pi)}, \quad \mathcal{K}_{n, j}^{(H)}=\frac{1}{12} \mathcal{K}_{j}^{(H)}, \quad \mathcal{K}_{n, j}^{(F)}=-\frac{1}{6} \mathcal{K}_{j}^{(F)}, \quad(j=2,3),
$$

while irreducible $2 \pi$-exchange leads to the expression

$$
\mathcal{K}_{n, 2}^{(2 \pi)}=\frac{m_{\pi}^{4}}{768 \pi^{2} f_{\pi}^{4}}\left\{I_{n}\left(\frac{p_{1}+p_{2}}{2 m_{\pi}}\right)-I_{n}\left(\frac{\left|p_{1}-p_{2}\right|}{2 m_{\pi}}\right)\right\},
$$




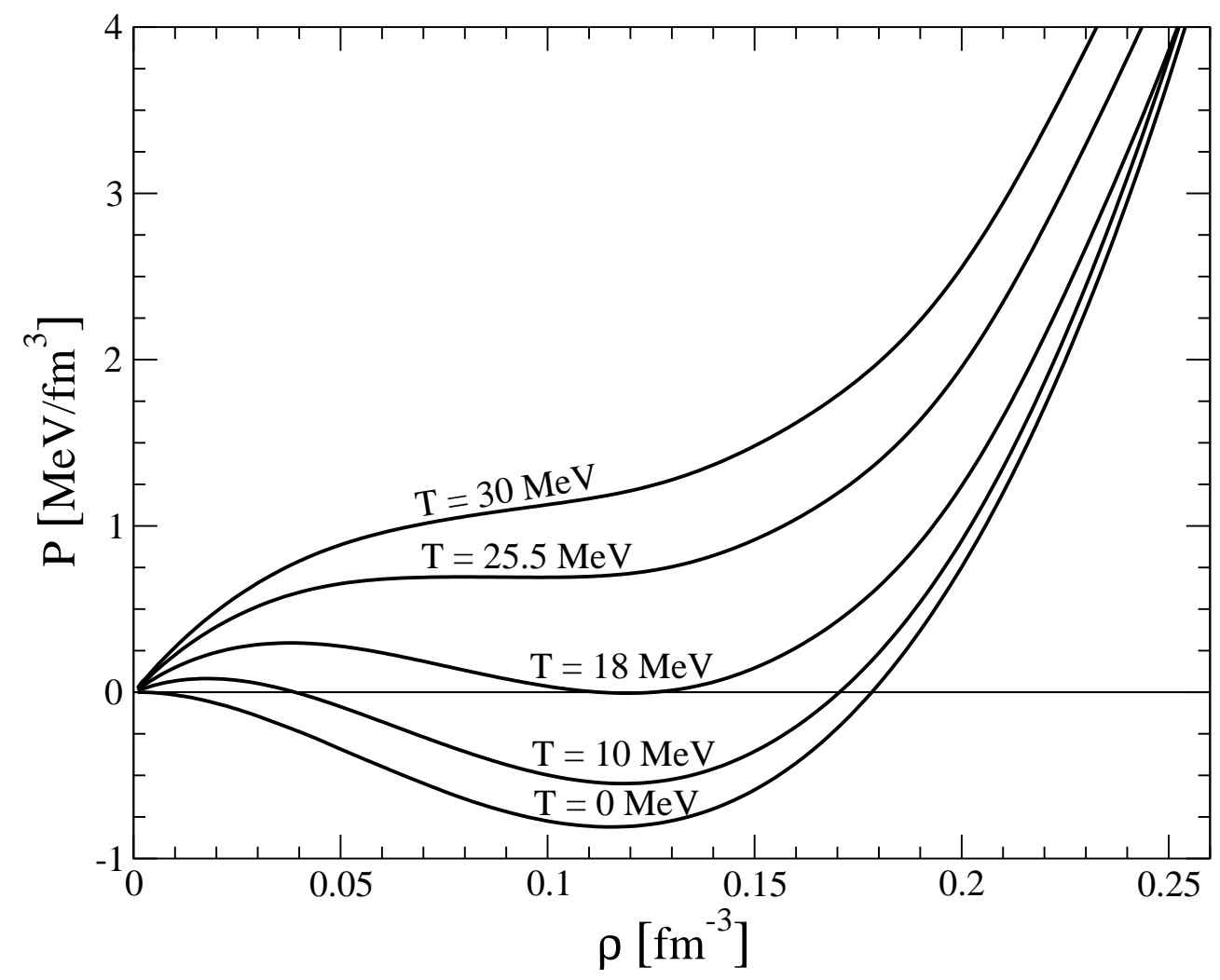

Figure 3: Pressure isotherms $P(\rho, T)$ of isospin symmetric nuclear matter. The coexistence region of the liquid and gas phase ends at the critical point $\rho_{c} \simeq 0.09 \mathrm{fm}^{-3}$ and $T_{c} \simeq 25.5 \mathrm{MeV}$.

with the modified function

$$
\begin{aligned}
I_{n}(x)= & 3\left(19 g_{A}^{4}-2 g_{A}^{2}-1\right) \ln ^{2}\left(x+\sqrt{1+x^{2}}\right) \\
& -2\left[g_{A}^{4}\left(26 x^{2}-7\right)+2 g_{A}^{2}\left(13+10 x^{2}\right)+5+2 x^{2}\right] x \sqrt{1+x^{2}} \ln \left(x+\sqrt{1+x^{2}}\right) \\
& +\left(19+118 g_{A}^{2}-257 g_{A}^{4}\right) x^{2}+\left(3+14 g_{A}^{2}-9 g_{A}^{4}\right) x^{4} \\
& +\left[12\left(1+6 g_{A}^{2}-15 g_{A}^{4}\right) x^{2}-4\left(13 g_{A}^{4}+10 g_{A}^{2}+1\right) x^{4}\right] \ln \frac{m_{\pi}}{2 \Lambda} .
\end{aligned}
$$

The expression for the power divergences specific to cut-off regularization changes accordingly in the case of pure neutron matter,

$$
\mathcal{K}_{n, 2}^{(\Lambda)}=-\frac{\Lambda p_{1} p_{2}}{64 \pi^{2} f_{\pi}^{4}}\left[2 g_{A}^{4} M+\left(3 g_{A}^{2}+1\right)\left(g_{A}^{2}-1\right) \Lambda\right] .
$$

Furthermore, for pure neutron matter the anomalous contribution gets reduced by a relative isospin factor $1 / 18$, or written in an explicit formula: $\overline{\mathcal{A}}_{n}\left(\rho_{n}, T\right)=\overline{\mathcal{A}}\left(2 \rho_{n}, T\right) / 9$ with $\overline{\mathcal{A}}(\rho, T)$ given in eq.(14). With this small weight factor the anomalous $2 \pi$-exchange Fock diagram becomes irrelevant for pure neutron matter.

In Fig. 4, we show by the full lines the calculated free energy per neutron $\bar{F}_{n}\left(\rho_{n}, T\right)$ for temperatures $T=10,20 \mathrm{MeV}$. The dashed lines in Fig. 4 correspond to the many-body calculation of the Urbana group [5]. These curves should be considered as a representative of the host of existing neutron matter calculations which scatter around them. In order to demonstrate the effects of the $n n$-interaction, we show by the dotted lines in Fig. 4 the result of a free neutron gas (as given by the one-body kernel $\mathcal{K}_{1}$ in eq.(18)). One observes a fair agreement of 


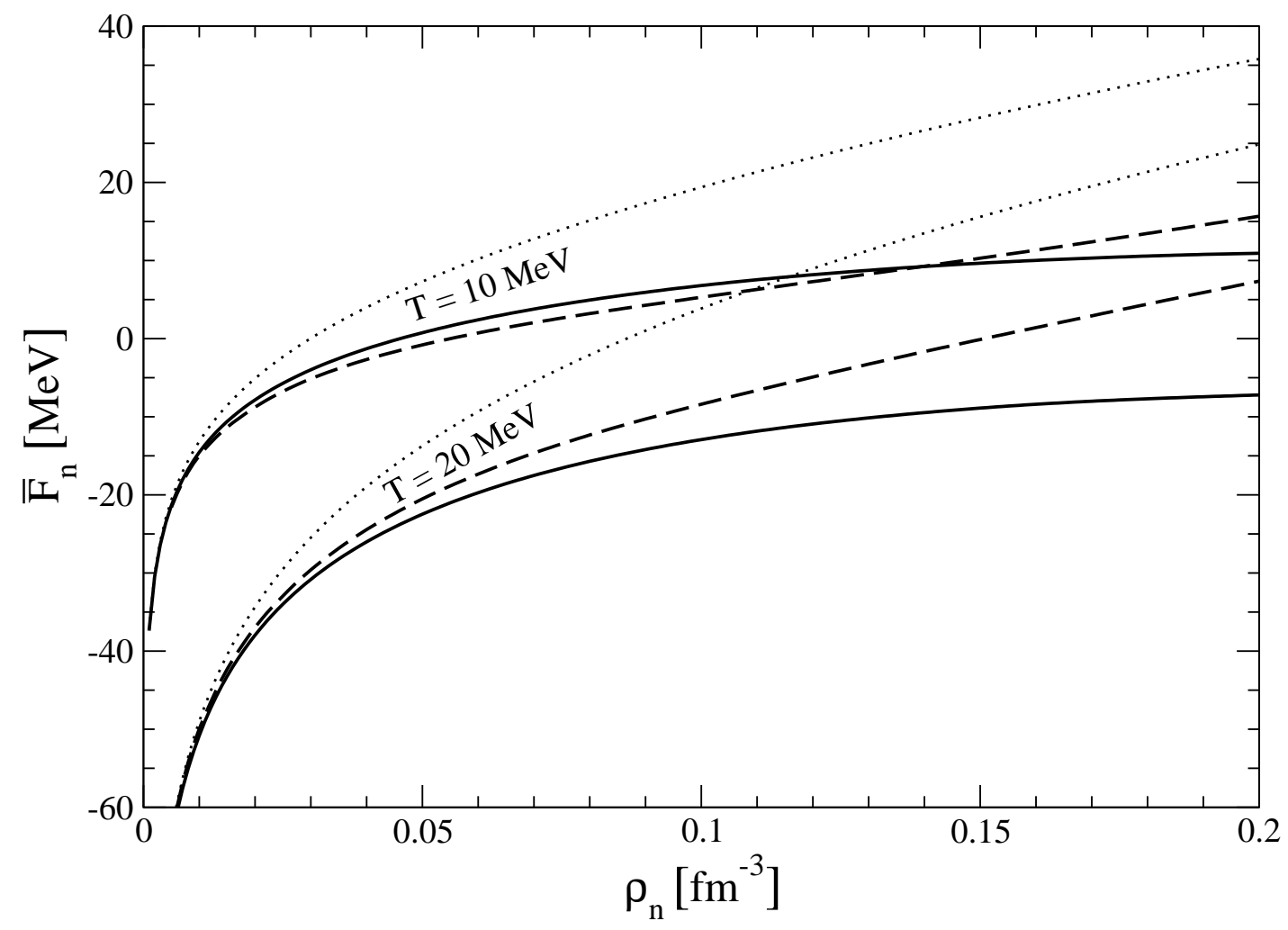

Figure 4: Free energy per particle of pure neutron matter $\bar{F}_{n}\left(\rho_{n}, T\right)$. The full lines give the result of chiral one- and two-pion exchange. The dashed lines correspond to the many-body calculation of ref. [5] and the dotted lines show the result for a non-interacting neutron gas.

our calculation with the results of ref.[5] for low neutron densities, $\rho_{n} \leq 0.2 \mathrm{fm}^{-3}$. One may also conclude from Fig. 4 that the parameterfree interaction effects generated by chiral $1 \pi$ - and $2 \pi$-exchange are fairly realistic in this low density region.

In summary, we have extended our recent three-loop calculation of nuclear matter in chiral perturbation theory to finite temperatures $T$. The contributions from one- and two-pion exchange diagrams are included in the density and temperature dependent free energy per particle $\bar{F}(\rho, T)$. This guarantees thermodynamical consistency and the correct $T=0$ limit to the energy per particle $\bar{E}\left(k_{f}\right)$. We reproduce the familiar liquid-gas phase transition of isospin symmetric nuclear matter. The predicted critical point, $T_{c}=25.5 \mathrm{MeV}, \rho_{c}=0.09 \mathrm{fm}^{-3}$ and $P\left(\rho_{c}, T_{c}\right)=0.69 \mathrm{MeV} \mathrm{fm}^{-3}$ lies however too high in temperature. The obvious reason for that is the strong momentum dependence of the single-particle potential $U\left(p, k_{f 0}\right)$ [8] near the Fermi-surface $p=k_{f 0}$ implying a huge effective nucleon mass $M^{*}\left(k_{f 0}\right) \simeq 3 M$. More elaborate calculations of nuclear matter in effective (chiral) field theory are necessary in order to cure this problem. Furthermore, we have considered pure neutron matter at finite temperatures $T$ in the same framework. We have found fair agreement with sophisticated many-body calculations for the low neutron densities, $\rho_{n} \leq 0.2 \mathrm{fm}^{-3}$, relevant for conventional nuclear physics.

\section{References}

[1] L.B. Csernai and J.I. Kapusta, Phys. Reports 131 (1986) 223; and refs. therein.

[2] D. Durand, E. Suraud and B. Tamain, "Nuclear Dynamics in the Nucleonic Regime", Institute of Physics Publishing, Bristol and Philadelphia, (2001); and refs. therein. 
[3] B.D. Serot and J.D. Walecka, Adv. Nucl. Phys. 16 (1986) 1; and refs. therein.

[4] J.I. Kapusta, "Finite-temperature Field Theory", Cambridge University Press, (1989), chapt. 10.

[5] B. Friedman and V.R. Pandharipande, Nucl. Phys. A361 (1981) 502.

[6] G. Sauer, H. Chandra and U. Mosel, Nucl. Phys. A264 (1976) 221.

[7] N. Kaiser, S. Fritsch and W. Weise, Nucl. Phys. A697 (2002) 255.

[8] N. Kaiser, S. Fritsch and W. Weise, Nucl. Phys. A700 (2002) 343.

[9] M. Lutz, B. Friman and C. Appel, Phys. Lett. B474 (2000) 7.

[10] A. Lejeune, P. Grange, M. Martzolff and J. Cugnon, Nucl. Phys. A453 (1986) 189.

[11] D.J. Thouless, "The Quantum Mechanics of Many-Body-Systems", Academic Press, New York and London, (1961), chapt.7.3.

[12] W. Kohn and J.M. Luttinger, Phys. Rev. 118 (1960) 41; J.M. Luttinger and J.C. Ward, Phys. Rev. 118 (1960) 1417. 